\title{
Magnetic fields in star-forming galaxies at high and low redshift
}

\author{
Timothy Garn*, Dominic Ford, Paul Alexander, David A. Green, Julia M. Riley \\ Astrophysics Group, Cavendish Laboratory, 19 J. J. Thomson Ave., Cambridge CB3 OHE, U.K. \\ E-mail: tsg25@cam.ac.uk
}

\begin{abstract}
As part of an ongoing series of deep GMRT surveys we have observed the Spitzer extragalactic First Look Survey field, producing the deepest wide-field 610-MHz survey published to date. We reach an rms noise level of $30 \mu \mathrm{Jy}$ beam $^{-1}$ before primary beam correction, with a resolution of $\sim 6 \operatorname{arcsec}$ over an area of $\sim 4 \mathrm{deg}^{2}$. By combining these observations with the existing 1.4-GHz VLA survey produced by Condon et al. (2003), along with infrared data in up to seven wavebands from the Spitzer Space Telescope, optical photometry from SDSS and a range of spectroscopic redshift surveys, we are able to study the relationship between radio luminosity and star formation rate in star-forming galaxies up to $z \sim 1$. The large amount of multi-wavelength data available allows $k$-corrections to be performed in the radio due to the knowledge of the radio spectral index, and in the infrared through the use of a semi-empirical radiative transfer model which models star-forming regions, warm dust surrounding these regions, and diffuse interstellar dust, taking into account the star formation rate, star formation history and hydrogen column density within each galaxy. A strong correlation is seen between radio luminosity and the infrared-derived star formation rates, which is best fit by a slightly non-linear power-law. We look for cosmic evolution in the comparative radio brightness of star-forming galaxies by searching for deviations away from the global relationship. Any such deviation would indicate a systematic variation in one or more of the properties controlling synchrotron radiation, in particular an increase in the magnetic field strengths of star-forming galaxies over time. The data shows no evidence for such an effect, suggesting that there has been little evolution in the magnetic fields of galaxies since $z \sim 1$.
\end{abstract}

From Planets to Dark Energy: the Modern Radio Universe

October 1-5 2007

The University of Manchester, UK

${ }^{*}$ Speaker. 


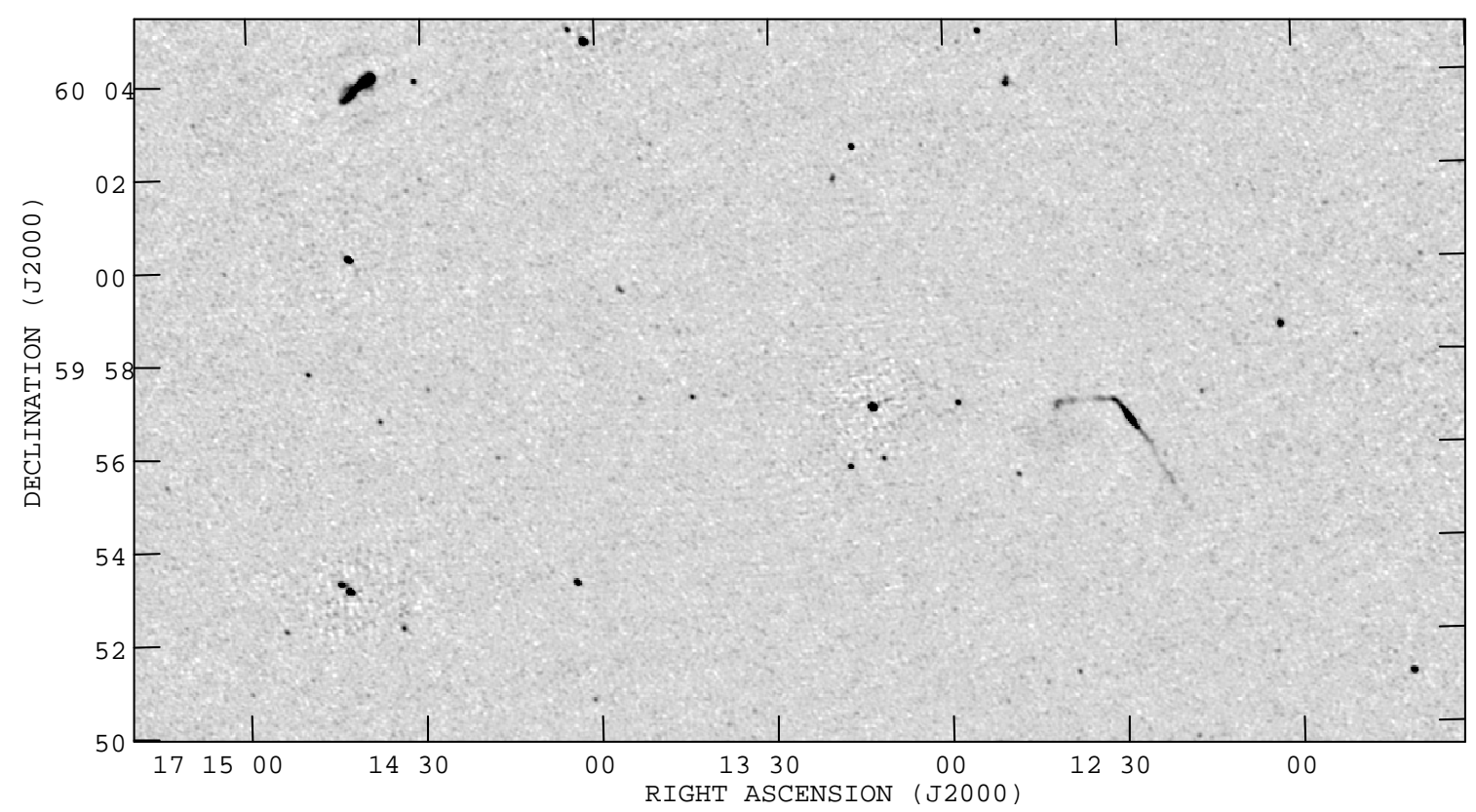

Figure 1: A sample section of the GMRT 610-MHz image of the Spitzer extragalactic First Look Survey field[1]. The grey-scale ranges between -0.2 and $1 \mathrm{mJy}^{\text {beam }}{ }^{-1}$.

\section{GMRT surveys}

We are carrying out a series of deep radio surveys with the Giant Metrewave Radio Telescope (GMRT) at $610 \mathrm{MHz}$, targeting regions with large amounts of existing multi-wavelength data. The first of these, the Spitzer extragalactic First Look Survey (xFLS) field[1] covers $\sim 4 \mathrm{deg}^{2}$ with a resolution of $\sim 6$ arcsec and rms noise level before primary beam correction of $30 \mu \mathrm{Jy} \mathrm{beam}^{-1}$. Fig. 1 shows a portion of the survey region, in order to demonstrate the image quality. This is the deepest wide-field GMRT survey published to date, with 3944 sources detected above a threshold of $5.25 \sigma$. Two other surveys have been completed, one covering $\sim 9 \mathrm{deg}^{2}$ of the ELAIS-N1 region[2] to a noise level of $70 \mu \mathrm{Jy}$ beam $^{-1}$, including a deeper region with $40 \mu \mathrm{Jy} \mathrm{beam}^{-1} \mathrm{rms}$, and another covering $\sim 5 \mathrm{deg}^{2}$ of the Lockman Hole[3] to a noise level of $60 \mu \mathrm{Jy} \mathrm{beam}^{-1}$. A survey of the ELAIS-N2 region is in progress, and will be completed in $2008^{1}$.

Our survey of the xFLS field was designed to complement the existing VLA 1.4-GHz survey[4], which has $\sim 5$ arcsec resolution and covers approximately the same area with a noise level of $23 \mu \mathrm{Jy}_{\text {beam }^{-1}}$. There is a great deal of complementary data available on the region - in particular, this was the location of the first of the deep surveys carried out by the Spitzer Space Telescope. We constructed a sample of 235 star-forming galaxies within the xFLS field, requiring all sources to have radio detections at $610 \mathrm{MHz}$ and $1.4 \mathrm{GHz}$, infrared detections at 24 and $70 \mu \mathrm{m}$ and a spectroscopic redshift. Where available, optical photometry from SDSS and further infrared data from the IRAC and MIPS instruments were included in the data set. More details on the sample selection procedure and the minimisation of potential sample biases can be found in [5].

\footnotetext{
${ }^{1} 610-\mathrm{MHz}$ images and source catalogues can be accessed via http://www. mrao.cam.ac.uk/surveys.
} 


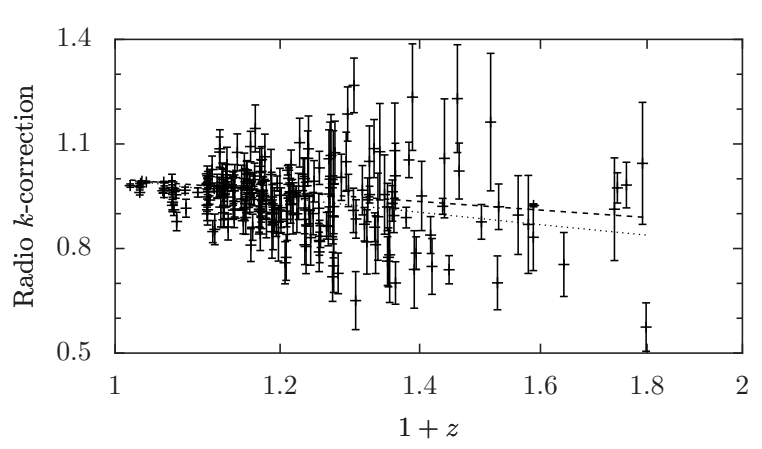

(a) Radio $k$-corrections.

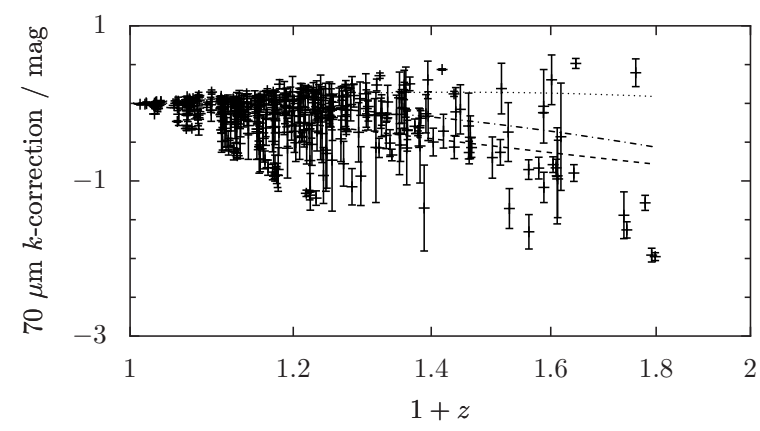

(b) Infrared $k$-corrections.

Figure 2: Radio $k$-corrections are shown in (a), calculated from the measured spectral index (points with error bars), $\alpha=0.7$ (dashed line) and $\alpha=0.8$ (dotted line). Infrared corrections are shown in (b), taken from the model spectra (points with error bars), M51 (dashed line), M82 (dotted) and Arp 220 (dash-dot).

\section{Radio and infrared $k$-corrections}

In order to study the luminosity of galaxies at high redshift it is necessary to $k$-correct the observed flux density $S_{v}$ at frequency $v$ to give a rest-frame luminosity $L_{v}$ at the same frequency. The dominant emission mechanism in the radio frequency range we are considering is synchrotron emission, which we assume to follow a power-law such that $S_{v}=S_{0} v^{-\alpha}$, and the luminosity of a source at redshift $z$ and luminosity-distance $d_{\mathrm{L}}$ is therefore given by $L_{v}=4 \pi d_{\mathrm{L}}^{2}(1+z)^{\alpha-1} S_{v}$. We assume a flat cosmology with $\Omega_{\Lambda}=0.74$ and $H_{0}=72 \mathrm{~km} \mathrm{~s}^{-1} \mathrm{Mpc}^{-1}$. Since radio observations were previously available at only one frequency, radio $k$-corrections carried out in the xFLS region by other authors have assumed a single spectral index for all sources (either 0.7 or 0.8 ). Using the two frequency data, we construct a spectral index distribution for our sources[5] and find a peak near 0.8 , but considerable spread. Fig. 2a shows our calculated radio $k$-corrections compared with those using a constant value for $\alpha$ of 0.7 or 0.8 ; using an assumed spectral index leads to a significant difference in $k$-correction for individual sources. In particular, the use of the observed spectral index permits $k$-corrections which are greater than unity.

Model optical-infrared spectral energy distributions (SEDs) were generated using the Starburst99 stellar spectral synthesis code[6], together with a semi-empirical radiative transfer model for the propagation of radiation through the dusty interstellar medium [5, 7]. The model is made up of star-forming regions, warm dust associated with these regions, and a diffuse distribution of cold dust throughout the galaxy. The star-formation rate (SFR), star formation history and dust mass were varied in order to create a grid of 2145 galaxy models, which were weighted according to their $\chi^{2}$ misfit to each source. From these, we calculate weighted $k$-corrections and SFRs for each galaxy. Previous works have typically taken local sources such as M82 to be representative of the whole star-forming galaxy population - however, the Malmquist bias dictates that the average luminosity of survey sources will increase with redshift, potentially making this assumption invalid. In Fig. $2 \mathrm{~b}$ we show the 70- $\mu \mathrm{m} k$-correction for all sources in our sample, along with those derived from three well-studied local sources (M51, M82 and Arp 220). The $k$-corrections derived from our model fitting follow the same general trends as for the local sources, but again the difference between individual galaxies and the local sources increases at higher redshift, by up to $\sim 1$ mag. 


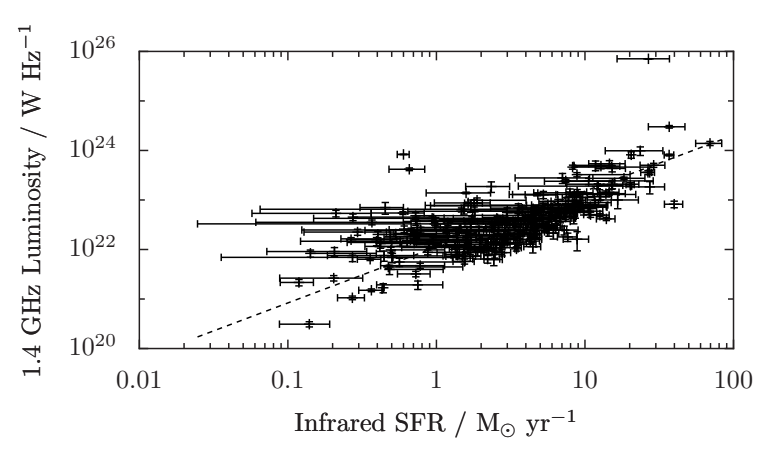

(a) 1.4-GHz luminosity against infrared SFR - the best-fit line is a power law given by Equation 3.1.

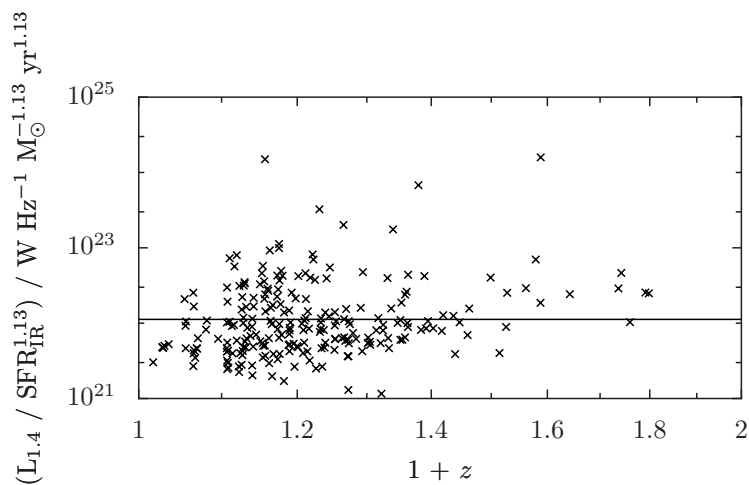

(b) The redshift dependence of the relationship seen in (a). The solid line represents the global value from Equation 3.1.

Figure 3: Comparison between radio luminosity and SFR (a), and the variation in this relationship with redshift (b).

\section{The relationship between star formation rate and radio luminosity}

The radio luminosity of a galaxy can be related to its current supernova rate, and therefore to its SFR about $30 \mathrm{Myr}$ earlier (the approx. lifetime of the least massive stars to form a supernova) through the use of an assumed Initial Mass Function. There are large uncertainties present in the normalisation between supernova rate and luminosity, and there is no a-priori reason to assume that the complex processes involved in synchrotron emission should lead to a linear relationship between supernova rate and radio luminosity. We therefore look directly at the relationship seen between $1.4 \mathrm{GHz}$ luminosity $L_{1.4}$ and SFR, seen in Fig. 3a, and find that the relationship is best fit by a power-law given by

$$
\log _{10}\left(L_{1.4}\right)=(22.05 \pm 0.04)+(1.13 \pm 0.07) \times \log _{10}(\mathrm{SFR}) .
$$

There are a few sources that are significantly more radio-bright than this relationship - these are likely to have some radio emission resulting from AGN activity as well as due to supernovae, since the AGN rejection in [5] was performed based upon optical spectroscopy alone. The quoted results remain consistent when these sources are removed from the analysis.

We probe the redshift dependence of the relationship between radio luminosity and SFR, by looking at deviations away from the global form found in Equation 3.1. This allows us to search for cosmic evolution in the comparative radio brightness of star-forming galaxies. Fig. 3b shows the redshift dependence of $L_{1.4} / \mathrm{SFR}^{1.13}$ - no significant variation is seen out to a redshift of 1 .

\section{Discussion \& Conclusions}

The large amount of multi-wavelength information available on regions such as the Spitzer extragalactic First Look Survey field mean that it is possible to create large samples of star-forming galaxies which contain a significant amount of spectral information. This allows individual $k$ corrections to be performed, using the knowledge of the optical stellar emission, infrared thermal dust emission and radio synchrotron emission from each galaxy in the sample. 
We find the relationship between radio luminosity and star formation rate has a slightly nonlinear form with power law index of $1.13 \pm 0.07$. By comparing the radio luminosity and infraredderived star formation rates of galaxies between $z \sim 1$ and the present day, we find no evidence for significant evolution in the radio luminosity of star-forming galaxies.

Any systematic variation over time in the radio luminosity of galaxies with particular SFR would imply an evolution in one or more of the properties that controls synchrotron radiation, and lead to the tight infrared / radio correlation[8]. In particular, an increase in the magnetic field strength of galaxies since redshift $1(\sim 7.7 \mathrm{Gyr})$ would lead to greater synchrotron emission from the electron population around supernova remnants and a greater chance of electron 'confinement' - electrons radiating away all of their energy within a galaxy, rather than escaping. This would lead to an increase in the radio luminosity for sources with given SFR at lower redshifts. No such effect is seen, suggesting that there has been little evolution in the magnetic fields of galaxies over this redshift range.

\section{Acknowledgments}

We thank the staff of the GMRT who have made these observations possible. The GMRT is operated by the National Centre for Radio Astrophysics of the Tata Institute of Fundamental Research, India. TG and DCF both thank the UK STFC for Studentships. This work has made use of the distributed computation grid of the University of Cambridge (CAMGRID).

\section{References}

[1] T. Garn, D. A. Green, S. E. G. Hales, J. M. Riley, P. Alexander, Deep 610-MHz Giant Metrewave Radio Telescope observations of the Spitzer extragalactic First Look Survey field - I. Observations, data analysis and source catalogue, MNRAS 376 (2007) 1251 [astro-ph / 0701534 ]

[2] T. Garn, D. A. Green, J. M. Riley, P. Alexander, A 610-MHz survey of the ELAIS-N1 field with the Giant Metrewave Radio Telescope - Observations, data analysis and source catalogue, MNRAS $\mathbf{3 8 3}$ (2008) 75 [astro-ph/0710.1500]

[3] T. Garn, D. A. Green, J. M. Riley, P. Alexander, A 610-MHz survey of the Lockman Hole with the Giant Metrewave Radio Telescope - I. Observations, data reduction and source catalogue for the central $5 \mathrm{deg}^{2}$, MNRAS, in press (2008) [astro-ph/0804.2421]

[4] J. J. Condon, W. D. Cotton, Q. F. Yin, D. L. Shupe, L. J. Storrie-Lombardi, G. Helou, B. T. Soifer, M. W. Werner, The SIRTF First-Look Survey. I. VLA Image and Source Catalog, AJ 125 (2003) 2411

[5] T. Garn, D. C. Ford, P. Alexander, Deep 610-MHz Giant Metrewave Radio Telescope observations of the Spitzer extragalactic First Look Survey field - II. The redshift evolution of the infrared / radio correlation, MNRAS, submitted (2008)

[6] C. Leitherer, et al., Starburst99: Synthesis Models for Galaxies with Active Star Formation, ApJS 123 (1999) 3 [astro-ph/9902334]

[7] D. C. Ford, B. Nikolic, P. Alexander, A Semi-Empirical Model of the Infrared Emission from Galaxies, MNRAS, in press, (2008) [astro-ph/0805.1732]

[8] P. N. Appleton, et al., The far-and mid-infrared/radio correlations in the Spitzer extragalactic First Look Survey, ApJS 154 (2004) 147 [astro-ph/ 0406030 ] 